\title{
Solution of Self-similar Equations of the k- $\varepsilon$ Model in the Shear Turbulent Mixing Problem and Its Numerical Simulation
}

\author{
Vyacheslav P. Statsenko, Yulia V. Tret'yachenko and Yury V. Yanilkin ${ }^{*}$ \\ Russian Federal Nuclear Centre, VNIIEF, Sarov, 607200, Russia
}

\begin{abstract}
The paper presents the k- $\varepsilon$ model equations of turbulence with a single set of constants chosen by the authors, which is appropriate to simulate a wide range of turbulent flows. The model validation has been performed for a number of flows and its main results are given in the paper. The turbulent mixing of flow with shear in the tangential velocity component is discussed in details. An analytical solution to the system of ordinary differential equations of the k- $\varepsilon$ model of turbulent mixing has been found for the self-similar regime of flow. The model coefficients were chosen using simulation results for some simplest turbulent flows. The solution can be used for the verification of codes. The numerical simulation of the problem has been performed by the 2D code EGAK using this model. A good agreement of the numerical simulation results with the self-similar solution, 3D DNS results and known experimental data has been achieved. This allows stating that the $\mathrm{k}-\varepsilon$ model constants chosen by the authors are acceptable for the considered flow.
\end{abstract}

Key words: The k- $\varepsilon$ model of turbulent mixing, shear turbulent mixing, self-similar equations, numerical simulation.

\section{Nomenclature}

If not additionally specified, the following notations are used in this paper:

$\begin{array}{ll}\mathrm{t} & \text { Time } \\ \mathrm{L} & \text { Length } \\ \mathrm{u} & \text { Mass velocities } \\ \rho & \text { Density } \\ \mathrm{e} & \text { Specific energy } \\ \mathrm{T} & \text { Temperature } \\ \mathrm{R} & \text { Reynolds stress tensor } \\ \mathrm{s} & \text { Specific entropy } \\ \alpha & \text { Mass fraction } \\ \mathrm{P} & \text { Pressure } \\ \mathrm{P}_{\mathrm{T}} & \text { Turbulent pressure } \\ \mathrm{k} & \text { Turbulent energy } \\ \varepsilon & \text { Turbulent energy dissipation rate } \\ \mathrm{D} & \text { Kinematic coefficient of } \\ \mathrm{G}_{1} & \text { Turbulent viscosity } \\ \mathrm{G}_{2} & \text { Shear generation of turbulence } \\ A \equiv\left(\rho_{2}-\rho_{1}\right) /\left(\rho_{2}+\rho_{1}\right) & \text { Aravitation generation of } \\ c_{\mu}, \sigma_{m}, \sigma_{h}, \sigma_{k}, & \text { Turbulence } \\ \sigma_{\varepsilon}, c_{\varepsilon 1}, c_{\varepsilon 2}, c_{\varepsilon 3} & \text { k- } \varepsilon \text { model coefficients } \\ & \end{array}$

\footnotetext{
"Corresponding author: Yury Vasilyevich Yanilkin, professor,
} research field: computational mathematics.

\section{Introduction}

The k- $\varepsilon$ model is one of the most successful models of turbulence and it is commonly used in engineering practice and research activities. Similarly to any other phenomenological model of turbulence, the k- $\varepsilon$ model has a set of semi-empirical coefficients. Usually, they can be found by solving the simplest self-similar problems, for which experimental data is available. In the self-similar stage, the effect of initial conditions, or some other factors introducing additional parameters to the description of flow is eliminated. The choice of model constants is made, as a rule, for a certain type of flows caused by the Rayleigh-Taylor, Kelvin-Helmholtz, or Richtmyer-Meshkov instabilities and they are different for various flows. However, in practice, it's not possible to vary model constants for different types of flows and there is a need in a single set of constants suitable for a wide range flows. So, a representative class of flows is required to select constants. Of course, the chosen constants, though it is universal to a sufficient extent, 
may be of a higher, or a lower accuracy (depending on each particular flow) in comparison with a specialized set of constants for a given flow. For the code in use and for the chosen set of constants, it is better to have a comprehensive range of test problems.

Paper [1] considers several simplest flows, for which both experimental data and 3D ILES results are available, and certain semi-empirical coefficients for the $\mathrm{k}-\varepsilon$ model implemented in the EGAK code [2] have been selected by comparing with this data. Some of these flows are considered in the present paper, the numerical simulation results have been also obtained using the standard set of constants.

One of such problems is the classic problem of shear mixing in a plane mixing layer. The problem was studied analytically, experimentally, numerically in many papers [3-11]. For the self-similar regime, this flow type was numerically studied by the authors with the 2D ILES [3] and 3D ILES [4] methods. However, the 3D ILES [5], 3D LES [12], and 3D DNS [13, 14] simulations on finer grids than those used in Ref. [4] demonstrated a significant spread in results and the necessity of studying this type of flows in details.

The principal objective of the paper is to justify the previously selected set of constants of the k- $\varepsilon$ model for the flow type of interest. The authors had found an exact solution to the $\mathrm{k}-\varepsilon$ model's self-similar equations using these constants and then the solution was used for the EGAK code verification (it may be also used for the verification of any other code). The second objective is to validate the $\mathrm{k}-\varepsilon$ model by comparing the solution found with the available experimental data and 3D ILES results. Additionally, correctness of the chosen model constants was confirmed by comparing with results of simulations using the standard set of model coefficients.

For ease of understanding by the readers, we also give an approximate solution to the problem from paper [1], for comparison. We performed a more correct post-processing of the available 3D ILES results and studied a broader range of experimental data [6-11].

\section{The Proposed k- $\varepsilon$ Model and Some Test Problems}

\subsection{Principal Equations of the $k-\varepsilon$ Model}

Consider weakly compressible flows with a negligible molecular viscosity (large Reynolds numbers). The CFD equations in the presence of turbulent mixing can be written as:

$$
\begin{gathered}
\partial \rho / \partial t+\operatorname{div}(\rho \vec{u})=0 \\
\frac{\partial}{\partial t}\left(\rho u_{i}\right)+\frac{\partial}{\partial x_{k}}\left(\rho u_{i} u_{k}\right)=-\frac{\partial P}{\partial x_{i}}+\frac{\partial \sigma_{i k T}}{\partial x_{k}}+g_{i} \rho
\end{gathered}
$$

where, $\vec{g}$ is the mass force (gravity) acceleration.

The Reynolds stress tensor, $\sigma_{i j T} \equiv-\bar{\rho} \cdot \overline{u_{i}^{\prime} u_{j}^{\prime}}$ is approximated, as usually:

$$
\sigma_{i j T}=\rho D\left(\frac{\partial u_{j}}{\partial x_{i}}+\frac{\partial u_{i}}{\partial x_{j}}-\frac{2}{3} \delta_{j i} \frac{\partial u_{k}}{\partial x_{k}}\right)-P_{T} \delta_{j i}
$$

Here, $P_{T}=2 / 3 \rho k$ is turbulent pressure, $k$ is turbulent energy. The kinematic coefficient of turbulent viscosity is:

$$
D=c_{\mu} k^{2} / \varepsilon
$$

where, $\varepsilon$ is the turbulent energy dissipation rate.

The equation of specific energy, $\mathrm{e}=\mathrm{e}(\rho, \mathrm{P})$ looks like $\frac{\partial}{\partial t}(\rho e)+\frac{\partial}{\partial x_{n}}\left(\rho u_{n} e-P a_{n}+Q_{n}^{T}\right)=\rho \varepsilon-P \frac{\partial u_{n}}{\partial x_{n}}-G_{2}(5)$ where, the molecular flow of heat is assumed to be negligibly small:

$$
\begin{aligned}
G_{2}=a_{k} \frac{\partial P}{\partial x_{k}}, \quad a_{i} & \equiv \frac{\overline{\rho^{\prime} u_{i}^{\prime}}}{\rho}=-\frac{D}{\rho \sigma_{h}}\left(\frac{\partial \rho}{\partial s}\right)_{P} \frac{\partial S}{\partial x_{k}} \\
Q_{n}^{T} & =-\frac{\rho D}{\sigma_{h}}\left(\frac{\partial e}{\partial s}\right)_{P} \frac{\partial S}{\partial x_{k}},
\end{aligned}
$$

$S$ is specific entropy. For an ideal gas with a constant value of $\gamma$, we can write:

$$
a_{k}=\frac{D}{\sigma_{h}}\left(\frac{1}{\gamma p} \frac{\partial P}{\partial x_{k}}-\frac{1}{\rho} \frac{\partial \rho}{x_{k}}\right) .
$$

The mass fraction equation for one of the two components looks like: 


$$
\begin{aligned}
& \frac{\partial}{\partial t}(\alpha \rho)+\frac{\partial}{\partial x_{k}}\left(\alpha \rho u_{k}\right)= \\
& \frac{\partial}{\partial x_{k}}\left(\frac{\rho D}{\sigma_{m}}\left(\frac{\partial \alpha}{\partial s}\right) \frac{\partial s}{\partial x_{k}}\right)
\end{aligned}
$$

Here, the molecular diffusion flow is also negligible.

The EOS (Equation of state) for a mixture of ideal gases has the form:

$$
P=R T \rho / \mu ; \quad \mu \equiv \alpha / \mu_{1}+(1-\alpha) / \mu_{2}
$$

Differential equations for the turbulent energy and dissipation rate look like:

$$
\begin{gathered}
\frac{\partial}{\partial t}(\rho k)+\frac{\partial}{\partial x_{k}}\left(\rho k u_{k}\right)= \\
\left(G_{1}+G_{2}\right)-\rho \varepsilon+\frac{\partial}{\partial x_{k}}\left(\frac{\rho D}{\sigma_{k}} \cdot \frac{\partial k}{\partial x_{k}}\right) \\
\frac{\partial}{\partial t}(\rho \varepsilon)+\frac{\partial}{\partial x_{k}}\left(\rho \varepsilon u_{k}\right)= \\
\frac{\varepsilon}{k}\left(c_{\varepsilon 1} G_{1}+c_{\varepsilon 3} G_{2}-c_{\varepsilon 2} \rho \varepsilon\right)+\frac{\partial}{\partial x_{k}}\left(\frac{\rho D}{\sigma_{\varepsilon}} \frac{\partial \varepsilon}{\partial x_{k}}\right)
\end{gathered}
$$

where, $\mathrm{G}_{1}$ is the shear generation of turbulence:

$$
G_{1}=\sigma_{j k T} \cdot \partial u_{j} / \partial x_{k} \text {. }
$$

Eqs. (1)-(11) contain phenomenological coefficients: $c_{\mu}, \sigma_{m}, \sigma_{h}, \sigma_{k}, \sigma_{\varepsilon}, c_{\varepsilon 1}, c_{\varepsilon 2}, c_{\varepsilon 3}$. Various authors use different values of these coefficients for particular flows (see, for example, [15-18]). However, note that there is a "standard" set of constants used in a number of papers [16-19]: $c_{\mu}=0.09, \sigma_{m}=\sigma_{h}=0.9, \sigma_{k}=1$, $\sigma_{\varepsilon}=1.3, c_{\varepsilon 1}=c_{\varepsilon 3}=1.44, c_{\varepsilon 2}=1.92$.

The values of constants selected for the EGAK code for the full set of the simplest flows discussed below with regard to the modern $3 \mathrm{D}$ simulations are, as follows: $\quad c_{\mu}=0.12, \quad \sigma_{k}=\sigma_{\varepsilon}=3 / 4, \quad c_{\varepsilon 1}=1.15$, $c_{\varepsilon 3}=1, \quad \sigma_{h}=\sigma_{m}=1 / 1.7, \quad c_{\varepsilon 2}=1.7$. They differ from the standard ones.

\subsection{Decay of Homogeneous Isotropic Turbulence}

It follows from Eqs. (10) and (11) for the given problem that

$$
\partial k / \partial t=-\varepsilon ; \quad \partial \varepsilon / \partial t=-c_{\varepsilon 2} \varepsilon^{2} / k
$$

hence,

$$
\varepsilon / \varepsilon_{0}=\left(k / k_{0}\right)^{c_{\varepsilon 2}}
$$

From Eqs. (12) and (13) we obtain:

$$
k / k_{0}=\left(t / t_{0}\right)^{-m} ; \quad t_{0} \equiv m k_{0} / \varepsilon_{0} .
$$

Here, $m=1 /\left(c_{\varepsilon 2}-1\right)$.

For the spatial scale of turbulence, $\Lambda=k^{3 / 2} / \varepsilon$ with regard to Eq. (14) we have:

$$
\begin{gathered}
\Lambda / \Lambda_{0}=\left(t / t_{0}\right)^{\delta} ; \\
\delta=1-m / 2 ; \quad \Lambda_{0} \equiv k_{0}^{3 / 2} / \varepsilon_{0}
\end{gathered}
$$

The decay law for homogeneous isotropic turbulence $-\mathrm{m}=10 / 7, \delta=2 / 7$ - following from the theoretic considerations in [20] is confirmed by results of experiments [21]. For the values of $c_{\varepsilon 2}$ used in the given paper the corresponding values of $m$ and $\delta$ are given in Table 1.

As we can see, these values for $c_{\varepsilon 2}=1.7$ agree with the data from [20], [21], while the values for $c_{\varepsilon 2}=1.92$ in the standard model [16-18] significantly differ.

\subsection{Neutrally Stratified Turbulent Boundary Layer}

This problem has a single velocity component, $u_{x}=u_{x}(y)$, where, $\mathrm{y}$ is the distance to a rigid wall. Then, $G_{2}=0, \Lambda \sim k^{3 / 2} / \varepsilon \sim y$ and a typical time scale is $\tau \sim \Lambda / u_{*} \sim y$, where the dynamic velocity is $\quad u_{*}^{2} \equiv D \partial u_{x} / \partial y=$ const and we take $\partial u_{x} / \partial y>0, \rho=1$ for definiteness. This means $\tau$ is small near the wall, i.e. the turbulent flow becomes steady-state. Eq. (2) gives $\partial \sigma_{x y} / \partial y=0$ for this case, or, with regard to Eq. (4),

$$
\sigma_{x y}=-D \partial u_{x} / \partial y \equiv-u_{*}^{2}=\text { const },
$$

this agrees with our previous assumption. $\partial u_{x} / \partial y \sim u_{*} / y$ in this case and, hence, $D \sim y$.

Table 1 Parameters $m$ and $\delta$ of the decaying homogeneous isotropic turbulence for different values of coefficient c 2 .

\begin{tabular}{lll}
\hline $\mathrm{c}_{\varepsilon 2}$ & $\mathrm{~m}$ & $\delta$ \\
\hline 1.92 & 1.087 & 0.4565 \\
1.7 & $10 / 7$ & $2 / 7$ \\
\hline
\end{tabular}


However, $D \sim \Lambda \sqrt{k}$ and, hence, $k=$ const near the wall. Then, it follows from Eqs. (10) and (12) that

$$
G_{1}=D\left(\partial u_{x} / \partial y\right)^{2}=\varepsilon \text {. }
$$

Eq. (11) can be written as

$$
\frac{\varepsilon^{2}}{k}\left(c_{\varepsilon 1}-c_{\varepsilon 2}\right)+\frac{\partial}{\partial y}\left(\frac{D}{\sigma_{\varepsilon}} \frac{\partial \varepsilon}{\partial y}\right)=0
$$

With regard to Eqs. (6) and (16), we have $\varepsilon / k=\sqrt{c_{\mu}} \cdot \partial u_{x} / \partial y$.

Thus, Eq. (17) can be written as

$$
C_{f} f^{2}=\frac{\partial^{2} \ln f}{\partial y^{2}}
$$

where,

$$
\begin{gathered}
f \equiv \partial u_{x} / \partial y ; \quad C_{f} \equiv\left(\kappa_{K} / u_{*}\right)^{2} ; . \\
\kappa_{K} \equiv c_{\mu}^{1 / 4} \sqrt{\left(c_{\varepsilon 2}-c_{\varepsilon 1}\right) \cdot \sigma_{\varepsilon}}
\end{gathered}
$$

The solution to Eq. (18) has the form

$$
u=u_{*} / \kappa_{K} \ln \left(y / h_{0}\right),
$$

where, $\mathrm{h}_{0}$ is either a height of irregularity, or a laminar sub-layer width. We see from Eq. (19) that $\kappa_{K}$ is Karman constant. Its values for different sets of empirical coefficients used in this paper are given in Table 2.

According to the experimental data from Ref. [22], $\kappa_{K}=0.4 \pm 0.04$. It is clear from Table 2 that both option 1 and option 2 satisfactorily agrees with this data.

\subsection{Gravitational Mixing of a Plane Interfacial Layer}

Consider two half-spaces separated at initial time by plane $\mathrm{z}=\mathrm{z}_{\mathrm{c}}=0$ and filled out with incompressible fluids (gases) at rest with densities $\rho_{1}=1$ and $\rho_{2}=n=3$. The gravitational acceleration, $\mathrm{g}_{\mathrm{z}}=-1 \equiv-\mathrm{g}$ is directed from a heavy material to a light material. The self-similar problem of gravitational turbulent mixing in the layer described above is considered. We think that "gravitational turbulent mixing" is a more appropriate term than "mixing by Rayleigh-Taylor instability", because in the nonlinear stage of the Rayleigh-Taylor instability development the shear instability is of an equal importance for the direct 3D numerical simulation.

The interface $z=z_{0}$ is at rest in the coordinate system in use. Introduce quantity $L_{t} \equiv z_{2}-z_{1}$, which is the TMZ width in direction $\mathrm{z}$ determined by points $z_{1}, z_{2}$ at which a small enough value $(\varepsilon \approx 0.01)$ of concentration disturbance is achieved: $\alpha_{2}\left(z_{1}\right)=\varepsilon$, $\alpha_{2}\left(z_{2}\right)=1-\varepsilon, \alpha_{2}$ is the mass fraction of material with the initial density $\rho_{2}=n$.

The problem of turbulent mixing under a constant gravity (constant acceleration) on a plane interface of two incompressible fluids (gases) was experimentally investigated and described in a number of papers see [23-27]. It turns out that quantity $n$ really does not influence the dimensionless quantity $\alpha_{b}$ characterizing the penetration of a light fluid (bubbles) into a heavy fluid: $z_{2}-z_{c}=\alpha_{b} \cdot A \cdot g \cdot t^{2}$. The authors of [26] obtained $\alpha_{\mathrm{b}}=0.078$. In some other experiments, the value of $\alpha_{b}$ is lower, for example, $\alpha_{b}=0.06-0.07$ in $[23,24]$. In the experiments described in [27] and in the later experiments $[28,29]$ the $\alpha_{b}$ values are far less.

The problem was numerically studied in a number of works [25, 30-35] by performing $2 \mathrm{D}$ and $3 \mathrm{D}$ simulations with the DNS method. Simulations using a sufficiently fine grid give noticeably lower TMZ growth rates than those in experiments [23-27]. As it is shown in [33], such discrepancy is owing to the fact that measurement results were processed with a large weight of the initial non-self-similar time interval.

Table 2 The values of $\kappa_{K}$ for two sets of empirical coefficients.

\begin{tabular}{llllll}
\hline Option No. & $c_{\mu}$ & $c_{\varepsilon 1}$ & $\sigma_{\varepsilon}$ & $c_{\varepsilon 2}$ & $\kappa_{K}$ \\
1 & 0.09 & 1.44 & 1.3 & 1.92 & 0.433 \\
2 & 0.12 & 1.15 & $3 / 4$ & 1.7 & 0.378 \\
\hline
\end{tabular}


Results of simulations with the EGAK code using the $k-\varepsilon$ model and various experimental and calculated data are given in Table 3. Note, first, that we performed data post-processing in a more correct fashion (similarly to the simulation result post-processing in $[32,33])$ using experimental data from [27] for $\mathrm{n}=2.83$ and obtained $\alpha_{b}=0.031 \div 0.033$ (instead of $\alpha_{b} \approx 0.05$ given in this work), which agrees better with 3D simulation results in [33-35]. Also, the EGAK results (option 2) approximately agree with them, while simulations with the standard coefficients (option 1) give us the $\alpha_{b}$ values, which are lower by an order of magnitude in comparison with experiments and 3D simulation results.

\subsection{Gravitational Mixing in a Light (Heavy) Plane} Layer

A light (heavy) plane layer of density $\rho_{1}$ and initial width $d$ surrounded by a fluid of density $\rho_{0}$ is in the gravity field with acceleration $g$. The self-similar solution is at $L_{t} / d \rightarrow \infty$ ( $L_{t}$ is the layer width at time $t$ ), for the light layer we find it in the form (for the heavy layer, variations are clear):

$$
\frac{\rho}{\rho_{0}}=1-\frac{d \varphi(\eta)}{L_{t}(t)}\left(1-\frac{\rho_{1}}{\rho_{0}}\right), \eta \equiv \frac{z-z_{d}}{L_{t}} .
$$

Quantity $b$ is defined according to Ref. [32]:

$$
b \equiv \frac{d \tilde{L}}{d t} ; \quad \tilde{L} \equiv \frac{L_{t}}{\sqrt{d g\left(1-\rho_{1} / \rho_{0}\right)}} \equiv \frac{L_{t}}{B} .
$$

Here,

$$
B \equiv \sqrt{d g\left(1-\rho_{1} / \rho_{0}\right)}
$$

The values of quantity $b$ in the self-similar regime in the EGAK code simulations with varying coefficients are given in Table 4. It is clear from data in Table 4 that the $b$ width measurement results [36] are closer to the option 2 results of simulations. At the same time, the option 1 simulations give the values of $b$ which are several times lower than the experimental results in Ref. [36].

\section{Analytical Solutions for the Self-Similar Regime of an Incompressible Shear Flow of Uniform Density}

\subsection{Principal Equations}

The flow of interest has only one velocity component, $u_{x}$ varying in the $y$ coordinate alone. The molecular viscosity is considered to be negligible. The flow density has constant value, $\rho=1$ everywhere. For this problem, equations from subsection 1.1 have the following form:

Table 3 The value of $\alpha_{b}$ in the problem of gravitational mixing of a plane interfacial layer, $A \approx 0.5$.

\begin{tabular}{llllllll}
\hline Option No. & $c_{\mu}$ & $c_{\varepsilon 3}$ & $\sigma_{\varepsilon}$ & $\sigma_{k}$ & $\sigma_{h}$ & $c_{\varepsilon 2}$ & $\alpha_{\mathrm{b}}$ \\
\hline 1 & 0.09 & 1.44 & 1.3 & 1 & 0.9 & 1.92 & 0.0019 \\
2 & 0.12 & 1 & $3 / 4$ & $3 / 4$ & $1 / 1.7$ & 1.7 & 0.0235 \\
\hline Experiments [26] & & & & & & 0.078 \\
Experiments [27] & & & & & $0.051 \pm 0.005$ \\
Experiments [28] & & & & & 0.04 \\
Experiments [29] & & & & & $0.03-0.04$ \\
3D simulations [33] & & & & & 0.028 \\
3D simulations [34] & & & & & $0.022-0.03$ \\
3D simulations [35] & & & & & & 0.027 \\
\hline
\end{tabular}

Table 4 The coefficients values and simulation results for the problem of mixing in a light layer.

\begin{tabular}{llllllll}
\hline Option No. & $c_{\mu}$ & $c_{\varepsilon 3}$ & $\sigma_{h}$ & $\sigma_{k}$ & $\sigma_{\varepsilon}$ & $c_{\varepsilon 2}$ & $b$ \\
\hline 1 & 0.09 & 1.44 & 0.9 & 1 & 1.3 & 1.92 & 0.1 \\
2 & 0.12 & 1 & $1 / 1.7$ & $3 / 4$ & $3 / 4$ & 1.7 & 0.36 \\
\hline Experiments [36] & & & & & & $0.35 \div 0.37$ \\
\hline
\end{tabular}


- Discontinuity equation (it is satisfied automatically in the given case)

$$
\partial u_{k} / \partial x_{k}=0
$$

- Equations of motion

$$
\partial u_{x} / \partial t=\partial \sigma_{x y} / \partial y
$$

The Reynolds stress tensor, $\sigma_{i j} \equiv-\bar{\rho} \cdot \overline{u_{i}^{\prime} u_{j}^{\prime}}$ is approximated, as usually:

$$
\sigma_{x y}=D \partial u_{x} / \partial y,
$$

where, $D=c_{D} k^{2} / \varepsilon$ is coefficient of turbulent viscosity, $k$ is turbulent energy, $\varepsilon$ is the turbulent energy dissipation rate

The turbulent energy equation looks like

$$
\frac{\partial k}{\partial t}=G_{1}-\varepsilon+\frac{\partial}{\partial y}\left(c_{k} D \frac{\partial k}{\partial y}\right)
$$

equation for the turbulent energy dissipation rate has the form:

$$
\frac{\partial \varepsilon}{\partial t}=\frac{\varepsilon}{k}\left(c_{\varepsilon 1} G_{1}-c_{\varepsilon 3} \varepsilon\right)+\frac{\partial}{\partial y}\left(c_{\varepsilon} D \frac{\partial \varepsilon}{\partial y}\right)
$$

Here, the shear generation of turbulence is

$$
G_{1}=\sigma_{j i} \partial u_{j} / \partial x_{i}=D\left(\partial u_{x} / \partial y\right)^{2}
$$

Eqs. (22)-(26) have coefficients $c_{\mu}=0.12$, $\sigma_{k}=\sigma_{\varepsilon}=3 / 4, \quad c_{\varepsilon 1}=1.15, \quad c_{\varepsilon 2}=1.7$.

\subsection{Equations of the $k$ - $\varepsilon$ Model for the Self-Similar} Regime of Shear Mixing in a Plane Interfacial Layer

The model considered here can be used to describe a self-similar shear flow, such as the flow occurring in the initial section of a mixing layer at the edge of a jet flowing out of a nozzle, when the ratio between the jet velocity, $u_{1}$ and the nozzle velocity relative to the surrounding medium, $u_{2}$ is close to 1 : $m \equiv u_{1} / u_{2} \approx 1$. Another example is a homogeneous flow from a spacer plate having velocities $u_{1}$ and $u_{2}$ relative to the plate (such experiments were used in Refs. [6-11] to study a plane layer of mixing).

In the coordinate system of fluid with a uniform density the problem becomes one-dimensional and unsteady, i.e. in the self-similar regime the plane mixing layer's width grows linearly with time in proportion to the velocity difference $u_{0}$ between the jet velocity and the surrounding medium velocity. (For the detailed information, see Appendix A)

Introduce self-similar variables:

$$
\begin{gathered}
u_{x} \equiv d x / d t=u_{0} \cdot f(\chi) / 2, \\
k=u_{0}^{2} \cdot E(\chi), D=u_{0}^{2} \cdot t \cdot d(\chi), \quad \chi \equiv y /\left(u_{0} t\right) .
\end{gathered}
$$

The boundary condition for the tangential velocity $u_{x}$ varying in the $y$ coordinate only is

$$
u_{x}=\left\{\begin{array}{c}
u_{0} / 2, \quad y \geq y_{2} \\
-u_{0} / 2, \quad y \leq y_{1}=-y_{2}
\end{array}\right.
$$

where, $u_{0}=u_{2}-u_{1}$.

Write Eqs. (22) and (23) for the self-similar regime assuming that $v \equiv f^{\prime}$ :

$$
\chi v+(d v)^{\prime}=0 .
$$

Write Eqs. (24) and (25) with regard to (26) for the self-similar regime:

$$
\chi E^{\prime}+d \cdot v^{2} / 4-c_{D} \mathrm{e}+c_{k}\left(d \cdot E^{\prime}\right)^{\prime}=0
$$

$$
\begin{gathered}
\mathrm{e}+\chi \cdot \mathrm{e}^{\prime}+\mathrm{e} \\
\cdot\left(c_{\varepsilon 1} \cdot d \cdot v^{2} / 4-c_{\varepsilon 3} \cdot c_{D} \cdot \mathrm{e}\right) / E+c_{\varepsilon} \cdot\left(d \cdot \mathrm{e}^{\prime}\right)^{\prime}=0 .
\end{gathered}
$$

Here,

$$
\mathrm{e} \equiv E^{2} / d .
$$

\subsection{An Approximate Solution}

To find an approximate solution, consider Eqs. (24) and (25) in the central part of TMZ (for $\chi=0$ ), where the first-order derivatives of quantities $k, \varepsilon, D$ become zeroes and write Eq. (24) for the self-similar regime in the form:

$$
d \cdot v^{2} / 4-c_{D} E^{2} / d+c_{k}\left(d \cdot E^{\prime}\right)^{\prime}=0
$$

Accept approximations

$$
\begin{gathered}
K=E / d \approx \text { const }, \\
v \approx \text { const } .
\end{gathered}
$$

Then, it flows from Eq. (29) that 


$$
d=\left(\chi_{1}^{2}-\chi^{2}\right) / 2 \text {, }
$$

and from Eq. (32) we obtain

$$
\begin{aligned}
E^{\prime \prime}=-K=\left(c_{D} K^{2}-v^{2} / 4\right) / c_{k}, \text { i.e. } \\
v=2 \sqrt{K\left(c_{D} K+c_{k}\right)}
\end{aligned}
$$

Eq. (25) for the self-similar regime, with regard to Eqs. (34)-(37), gives us

$$
K=\frac{1-c_{\varepsilon}+c_{\varepsilon 1} c_{k}}{c_{D}\left(c_{\varepsilon 3}-c_{\varepsilon 1}\right)}
$$

Take $\mathrm{f}\left(\chi_{2}\right)=1 ; \quad \mathrm{f}\left(\chi_{1}\right)=-1$, and $\mathrm{L}=\mathrm{y}_{2}-\mathrm{y}_{1}$ (TMZ width) and obtain

$$
\begin{gathered}
v=\frac{d f}{d \chi} \approx \frac{2 A_{v}}{\chi_{2}-\chi_{1}}=\frac{A_{v}}{\left|\chi_{1}\right|}, \\
\hat{\dot{L}} \equiv \frac{1}{u_{0}} \cdot \frac{d L}{d t}=\chi_{2}-\chi_{1}=\frac{2 A_{v}}{v}=\frac{A_{v}}{\sqrt{K\left(c_{D} K+c_{k}\right)}},
\end{gathered}
$$

where, the correction coefficient, $A_{v}>1$ takes into account that the value of $v=f / d \chi \approx 2 A_{v} /\left(\chi_{2}-\chi_{1}\right)$ at the TMZ center (which is considered here) exceeds the average value of this quantity in TMZ, $\bar{v}=\overline{d f / d \chi}=2 /\left(\chi_{2}-\chi_{1}\right)$.

It follows from Eqs. (34) and (36) that the $E$ maximum is $E_{m}=K \chi_{1}^{2} / 2$ and with regard to Eq. (40) it allows finding the reduced turbulent energy maximum.

$$
E_{m}=\frac{K A_{v}^{2}}{2 v^{2}}=\frac{A_{v}^{2}}{8\left(c_{D} K+c_{k}\right)} .
$$

A more complicated approximate analytical solution to the $k-\varepsilon$ model equations, when $v$ is a function of $\chi$, is given in Ref. [19].

\subsection{Solution of Self-Similar Equations}

Write the second-order derivatives using Eqs. (30) and (31):

$$
\begin{gathered}
E^{\prime \prime}=\frac{c_{D} \mathrm{e}-\chi E^{\prime}-d \cdot v^{2} / 4-c_{k} d^{\prime} \cdot E^{\prime}}{c_{k} d} \\
\mathrm{e}^{\prime \prime}=\frac{\frac{\mathrm{e}}{E}\left(c_{\varepsilon 3} c_{D} \mathrm{e}-\frac{c_{\varepsilon 1}}{4} d \cdot v^{2}\right)-\mathrm{e}-\chi \mathrm{e}^{\prime}-c_{\varepsilon} d^{\prime} \cdot \mathrm{e}^{\prime}}{c_{\varepsilon} d}
\end{gathered}
$$

Solutions to Eqs. (29), (42) and (43) can be found numerically in the two ways: by integrating these equations in intervals $\chi=0$ to $\chi=\chi_{2}$ and $\chi=\chi_{1}$ to $\chi=0$. Consider the first way of integrating.

The boundary condition at point $\chi=0$ is

$$
E^{\prime}=d^{\prime}=0 \text {. }
$$

It also follows from Eqs. (45) and (30) that $v^{\prime}=\mathrm{e}^{\prime}=0$.

At the same point, set nonzero values of quantities (the approximate analytical solution is taken for the first approximation):

$$
v=v_{0}>0, \quad d=d_{0}>0, \quad E=E_{0}>0 .
$$

And, according to Eqs. (42) and (43), the second-order derivatives are:

$$
\begin{gathered}
E_{0}^{\prime \prime}=\frac{c_{D} \mathrm{e}_{0}-d_{0} v_{0}^{2} / 4}{c_{k} d_{0}}, \\
\mathrm{e}_{0}^{\prime \prime}=\frac{\frac{\mathrm{e}_{0}}{E_{0}}\left(c_{\varepsilon 3} c_{D} \mathrm{e}_{0}-\frac{c_{\varepsilon 1}}{4} d_{0} v_{0}^{2}\right)-\mathrm{e}_{0}}{c_{\varepsilon} d_{0}} .
\end{gathered}
$$

Integrate Eqs. (29), (42) and (43) between point $\chi=0$ and point $\chi=\chi_{2}$, where the values of quantities $d$ and $E$ are $d\left(\chi_{2}\right)=E\left(\chi_{2}\right)=0$. During the integration find $d$ using Eq. (32) and then find $v$ using the solution to Eq. (29)

$$
v=\frac{d_{0} v_{0}}{d} \cdot \exp \left\{-\int_{0}^{\chi} \frac{\chi}{d} \cdot d \chi\right\} .
$$

Vary the values of quantities in (45) to achieve zero values of $d$ and $E$ at the same time. In general, one more boundary condition following from (27), (28) is not satisfied in this case $f\left(\chi_{2}\right)=1$. However, it is easy to show that for the two solutions with different values $\chi=\chi_{2}$ and $\tilde{\chi}=\tilde{\chi}_{2}$ the scale transformation takes place:

$$
\chi=\frac{\tilde{\chi}}{\Delta}, v=\tilde{v}, f=\frac{\tilde{f}}{\Delta}, d=\frac{\tilde{d}}{\Delta^{2}}, E=\frac{\tilde{E}}{\Delta^{2}},
$$

where, $\Delta=\int_{0}^{\tilde{\chi}_{2}} \tilde{v} \cdot d \tilde{\chi}=\tilde{f}\left(\tilde{\chi}_{2}\right)$.

Thus, use solution $\tilde{v}, \tilde{d}, \tilde{E}$ giving us $\tilde{\chi}=\tilde{\chi}_{2}$, under which $\tilde{f}\left(\tilde{\chi}_{2}\right)=\Delta \neq 1$, and by making transformation (46) obtain the solution satisfying the 
condition $f\left(\chi_{2}\right)=1$. For this purpose, vary the values of $v_{0}$ and ratio $d_{0} / E_{0}$, which do not change during the transformation (46).

\section{Numerical Simulation Using the k- $\varepsilon$ Model}

\subsection{Problem Setting}

A planar interface of two incompressible fluids has a tangential velocity discontinuity, $\Delta u_{x} \equiv u_{0}=1$ (Fig. 1): above the interface corresponding to coordinate $y=1$, the fluid (gas) moves parallel to the interface with the velocity $u_{x}=u_{0} / 2$ and below the interface its velocity is $u_{x}=-u_{0} / 2$. The velocity on the interface is $u_{x}=0$. Boundary conditions on the left and on the right of the computational domain are periodic, the top and bottom boundaries are "rigid walls". Initially, the both domains are filled with ideal gases with $\gamma=1.4$ and density $\rho=1$ under pressure $P_{0}$ $=10$, i.e. the flow is close to the incompressible one. To check such conditions for sufficiency, simulations with pressure $\mathrm{P}_{0}=50$ were also performed using a grid of $\mathrm{N}=400$ points ( $\mathrm{N}$ is the number of points in domain along $y$ axis). The initial values of turbulence quantities, $k=0.001$ and $\varepsilon=0.025$, are set at the interface (in one layer of cells on each side of the interface). In all other cells, the initial values of these quantities are $k=\varepsilon=10^{-11}$.

Simulations were performed using a square Eulerian grid by varying the number of cells along $y$ axis (see Table 5).

4.2 Results of the 2D Simulations, Comparison with the Solutions of Self-Similar Equations and Measurements

The resulting profiles of the solutions described in subsection 3.4 are shown in Figs. 2, 3, 6 and 8. In simulations we used $N_{\chi}=4 \times 10^{4}$ cells within the range from the TMZ center to the boundary. The initial values of quantities in simulations were $v_{0}=$ $15.7675, \mathrm{E}_{0}=0.042, \mathrm{~d}_{0}=0.0023$.

As one can see from Fig. 2, the discrepancy in profiles is insignificant: both $2 \mathrm{D}$ simulations nearly coincide, which indicates that they converge with respect to the number of computational cells, and that the flow is sufficiently close to incompressible. The same is valid for the turbulent energy (Fig. 3): an insignificant discrepancy between solution in subsection 2.4 and results of the both 2D simulations (which coincide) is observed.

Eq. (28) should be written in the following form:

$$
D=u_{0}^{2} \cdot\left(t-t_{0}\right) \cdot d(\chi), \quad \chi \equiv \frac{y}{u_{0} \cdot\left(t-t_{0}\right)} .
$$

Here, $t_{0}$ is the intersection point of $x$ axis and the linear (self-similar) section of dependence $\hat{b}(t)=z_{0.9}(t)-z_{0.1}(t)$ (the line of black dots in Fig. 4a). According to data in Fig. $4 \mathrm{a}, t_{0}=-0.064$ for $\mathrm{N}=$ 400 and $\mathrm{N}=1000$, and $t_{0}=0.285$ for $\mathrm{N}=200$.

Note that there is almost no difference between the results in simulations with $\mathrm{N}=400$ and $\mathrm{N}=1000$. The turbulent energy maximum in TMZ as a function of time demonstrates the same behavior: we can see in Fig. $4 \mathrm{~b}$ that it quickly achieves one and the same constant value in all simulations.

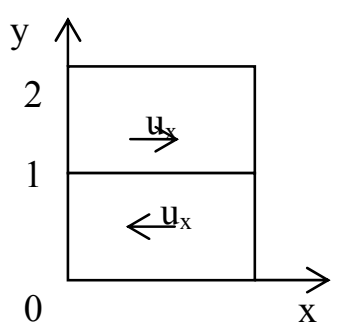

Fig. 1 Initial problem geometry.

Table 5 Options of simulations.

\begin{tabular}{lllll}
\hline Number of cells along $y$ axis & $\mathrm{N}=200$ & $\mathrm{~N}=400$ & $\mathrm{~N}=400$ & $\mathrm{~N}=1000$ \\
\hline Size of cell & $\mathrm{h}=0.01$ & $\mathrm{~h}=0.005$ & $\mathrm{~h}=0.005$ & $\mathrm{~h}=0.002$ \\
Pressure $\left(\mathrm{P}_{0}\right)$ & 10 & 10 & 50 & 10 \\
\hline
\end{tabular}




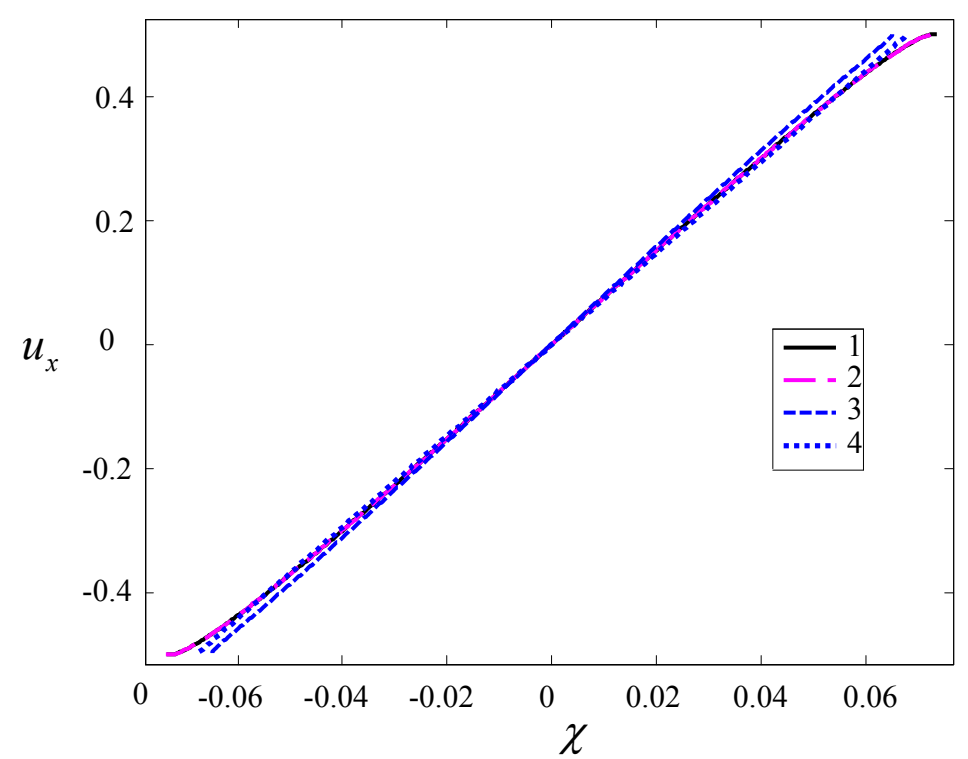

Fig. 2 The velocity profiles in $2 \mathrm{D}$ simulations: $1-\mathrm{N}=1000, \mathrm{P}_{0}=10 ; 2-\mathrm{N}=400, \mathrm{P}_{0}=50 ; 3$-solution in subsection 3.4; 4 -approximate solution.

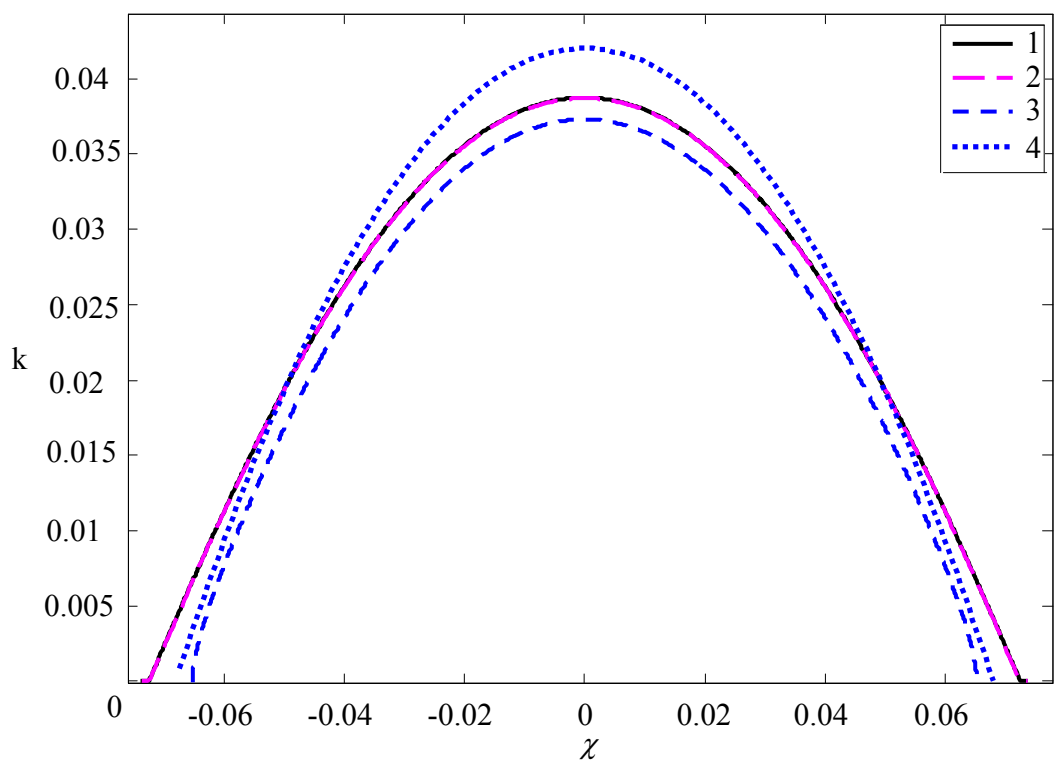

Fig. 3 The turbulent energy profiles. $2 \mathrm{D}$ simulations: $1-\mathrm{N}=1000, \mathrm{P}_{0}=10 ; 2-\mathrm{N}=400, \mathrm{P}_{0}=50 ; 3-$ solution 3.4; 4-approximate solution.

Fig. 5a illustrates function $F_{\varepsilon} \equiv \varepsilon_{m} \cdot\left(t-t_{0}\right)$ of the TMZ-maximum value $\varepsilon_{m}$ of the turbulent energy dissipation rate. This function also quickly achieves one and the same constant value. Function $F_{D} \equiv D_{m} /\left(t-t_{0}\right)$ of the TMZ-maximum value $D_{m}$ of the turbulent viscosity coefficient is shown in Fig. $5 b$. This function also quickly achieves its constant value, which is almost the same in simulations with various $\mathrm{N}$.
It follows from the analysis of this data that we observe convergence of the $2 \mathrm{D}$ simulation results obtained by varying the computational grid in use. Simulation results for grid $\mathrm{N}=200$ insignificantly differ from those for grids $\mathrm{N}=400$ and $\mathrm{N}=1000$ (which, in turn, are actually indistinguishable).

We see in Fig. 6 that there is an insignificant discrepancy between the turbulent viscosity coefficient profile calculated with the method in subsection 3.4 


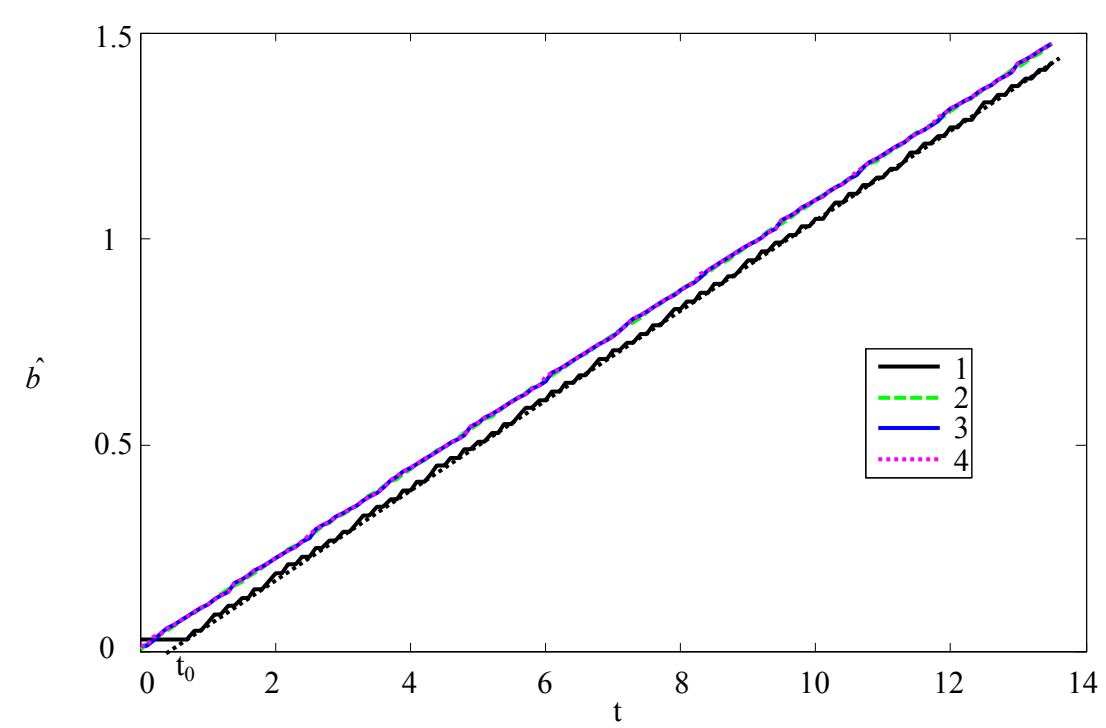

(a)

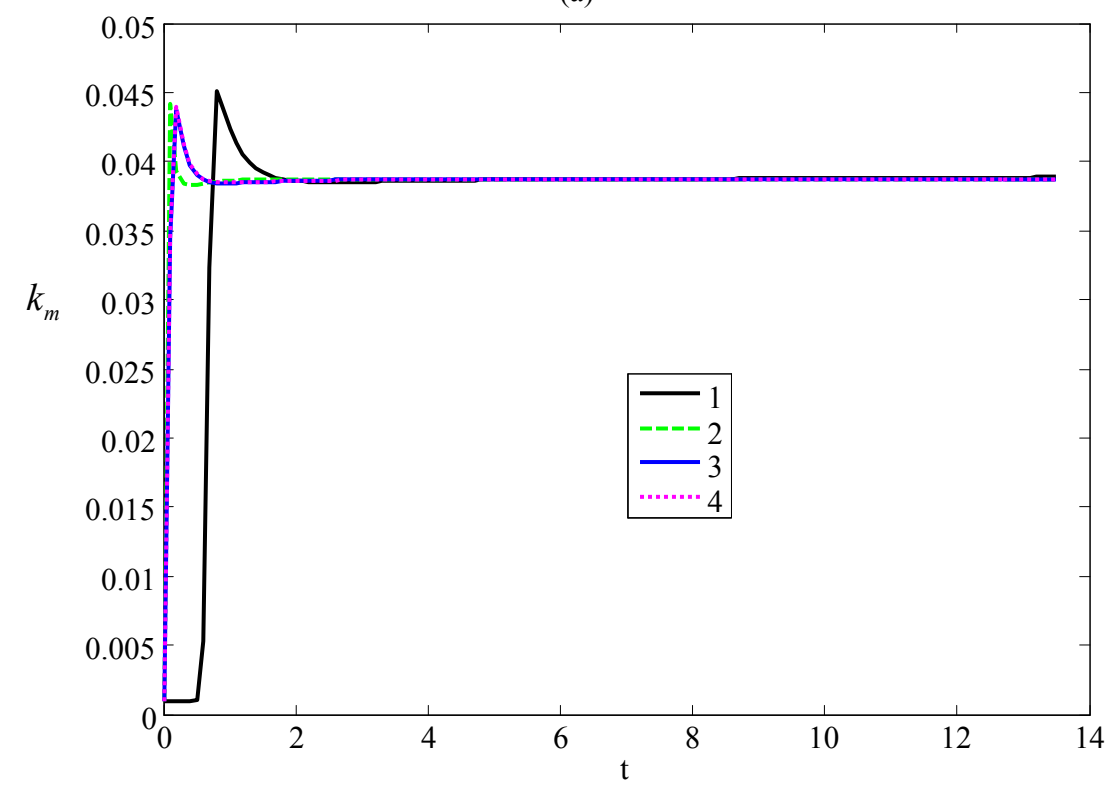

(b)

Fig. 4 The turbulent quantities vs time in 2D simulations: (a) TMZ width $\hat{b}$, (b) turbulent energy maximum in TMZ: 1 $P_{0}=10, N=200 ; 2-P_{0}=10, N=1000 ; 3-P_{0}=10, N=400 ; 4-P_{0}=50, N=400$.

and profiles in both $2 \mathrm{D}$ simulations (they coincide). There is a satisfactory agreement, in general, with the approximate analytical solution.

In solution 3.4 at the right boundary, both the flux of turbulent energy dissipation rate, $\tilde{\varepsilon} \equiv c_{D} \cdot \mathrm{e}$ and the flux of turbulent energy itself become zeroes (Fig. 7). There is an insignificant discrepancy between the profiles of turbulent energy dissipation rate in solution 3.4 and in 2D simulations (which are almost coincident) (Fig. 8).

The integrated data obtained by solving the system of ordinary differential Eqs. (30)-(33) describing the self-similar regime of shear flow are as follows:

from subsection 3.4:

$$
\begin{gathered}
\beta=b /\left(u_{0} \cdot t\right) \equiv \Delta \chi_{b}= \\
0.1105, k_{m}=\max (k)=0.0373 .
\end{gathered}
$$

Here, according to the definition in Refs. [9, 10], $b=z_{0.95}-z_{0.1}$ is the TMZ width found for level 


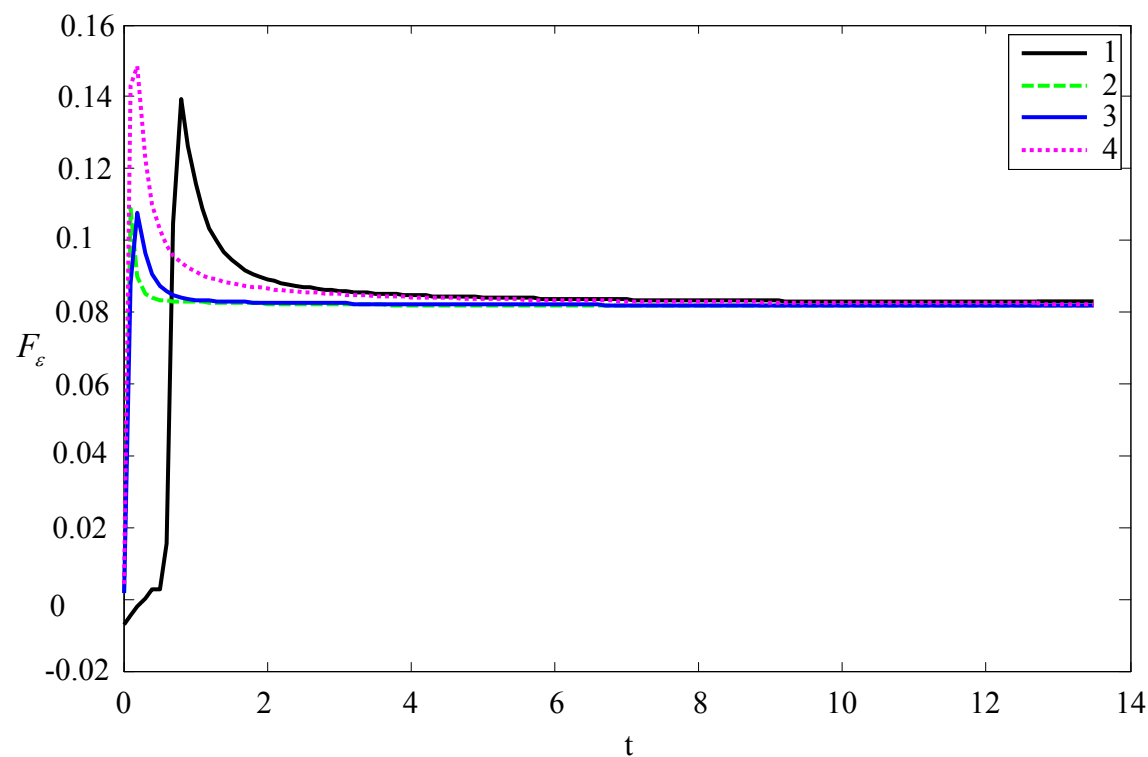

(a)

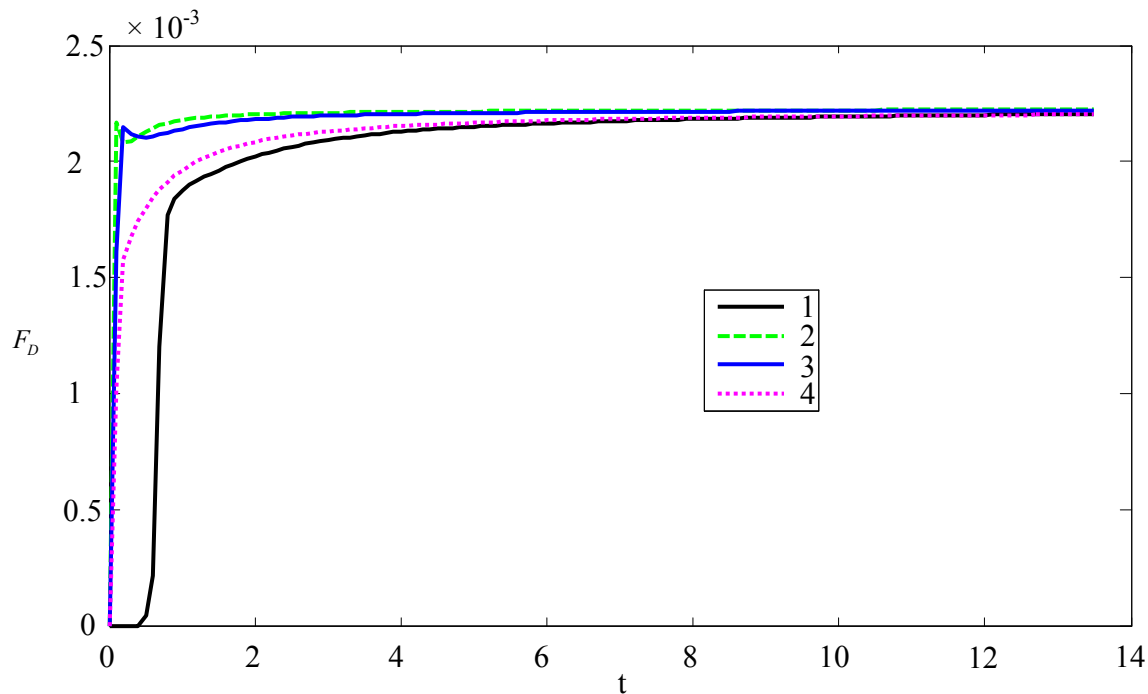

(b)

Fig. 5 TMZ-maximum value of turbulent quantities vs time in 2D simulations: (a) the function of turbulent energy dissipation rate, (b) the turbulent viscosity coefficient function: $1-P_{0}=10, N=200 ; 2-P_{0}=10, N=1000 ; 3-P_{0}=10, N=400$. $4-\mathbf{P}_{0}=\mathbf{5 0}, \mathbf{N}=\mathbf{4 0 0}$.

and

$$
\frac{u_{x}}{u_{0}}\left(z_{0.95}\right)=\frac{u_{x}}{u_{0}}\left(\chi_{0.45}\right)=0.45
$$

$$
\frac{u_{x}}{u_{0}}\left(z_{0.1}\right)=\frac{u_{x}}{u_{0}}\left(\chi_{-0.4}\right)=-0.4,
$$

or in the spacer plate's coordinate system:

$$
\frac{\tilde{u}_{x}}{u_{0}}\left(z_{0.95}\right)=0.95 \text { and } \frac{\tilde{u}_{x}}{u_{0}}\left(z_{0.1}\right)=0.1 \text {. }
$$

The values in Eq. (47) are close both to the results of $2 \mathrm{D}$ simulations with the $\mathrm{k}-\varepsilon$ model $(\beta=0.1154$,

$\left.k_{m}=0.0388\right)$ and results of the approximate analytical solution $\left(\beta=0.1156, k_{m}=0.042\right)$. The $\beta$ value in (47) also agrees with the 3D ILES results in $[4,5], 3 \mathrm{D}$ LES results in [12] and 3D DNS results in $[13,14]$ (see Appendix A) and measurement results in [9] (see Appendix B). The maximum values of turbulent energy in all the 2D simulations are close and do not contradict the available measurement results. 


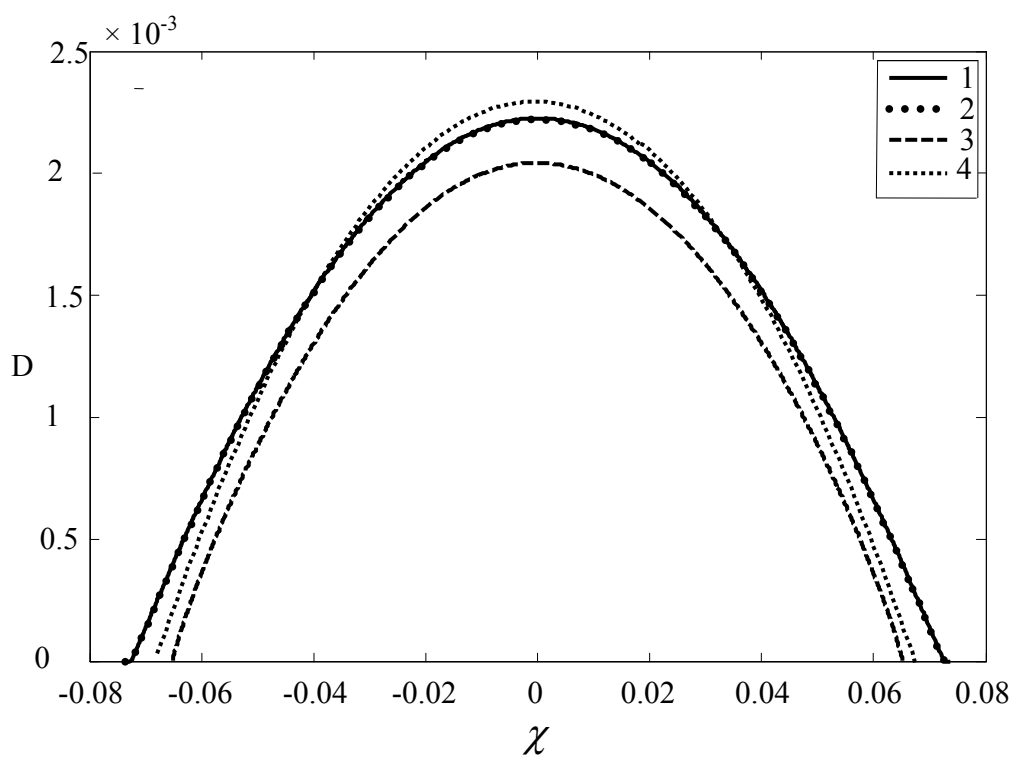

Fig. 6 The turbulent viscosity coefficient profiles in $2 D$ simulations: $1-N=1000, P_{0}=10 ; 2-N=400, P_{0}=50 ; 3-$ solution 3.4; 4 - approximate analytical solution.

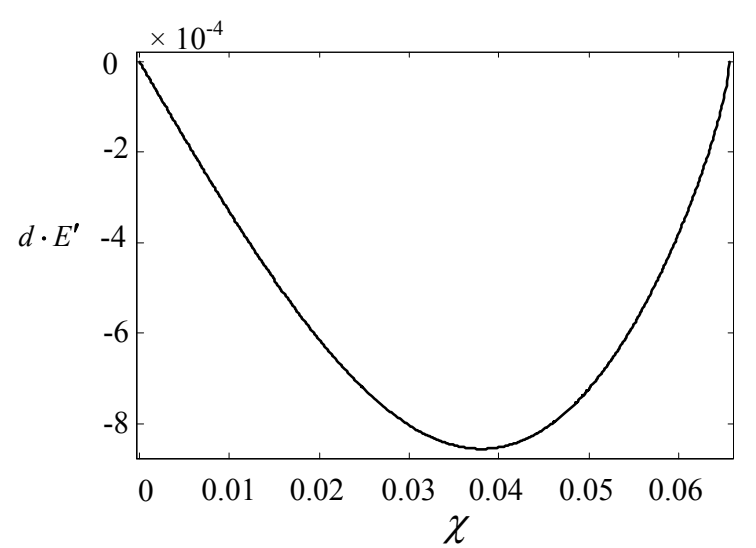

(a)

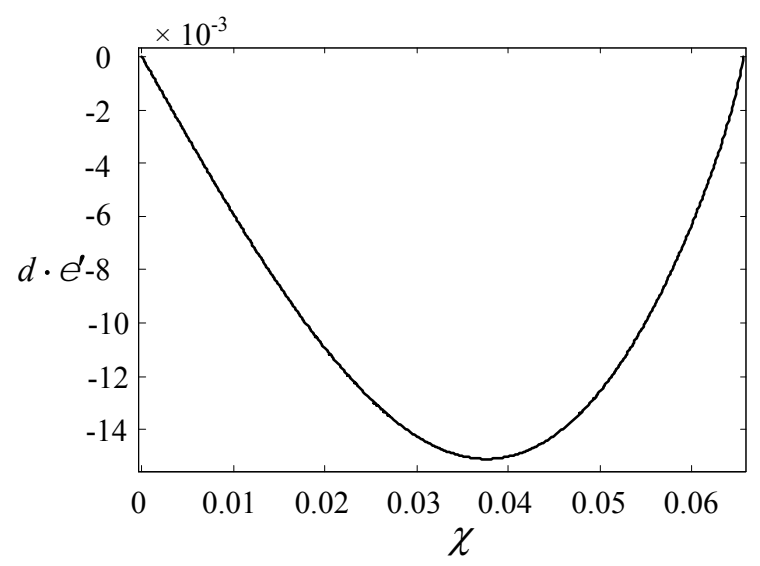

(b)

Fig. 7 Profiles of the turbulent energy flux (left) and dissipation rate flux (right) in solution 3.4.

Thus, the velocity profiles in 2D simulations satisfactorily agree with results of the numerical integration of ordinary differential equations (29)-(32) describing the self-similar regime of shear flow and with the approximate analytical solution, as it is clearly seen in Figs. 2, 3, 6 and 8.

\section{Discussion of results and conclusions}

The 2D EGAK code results of the numerical simulation using the $\mathrm{k}-\varepsilon$ model demonstrate quick transition to the self-similar regime, the initial values of $k$ and $\varepsilon$ are forgotten. This regime is characterized by a linear growth of TMZ width with time and by time-constant maxima of turbulent energy in TMZ $\left(k_{m}\right)$ and functions of its dissipation rate $\left(\varepsilon_{m}\right)$, as well as the turbulent viscosity coefficient $\left(D_{m}\right): \varepsilon_{m}\left(t-t_{0}\right)$ and $D_{m} / t$.

A system of ordinary differential equations has been constructed for the self-similar regime of shear flow and the equation system solution methods are presented. The solutions found are in a good agreement with the 2D EGAK code simulation results. Thus, they can be used for the code verification.

The obtained solutions agree with the approximate analytical solution and, hence, it can be used for the preliminary selection of the k- $\varepsilon$ model coefficients 


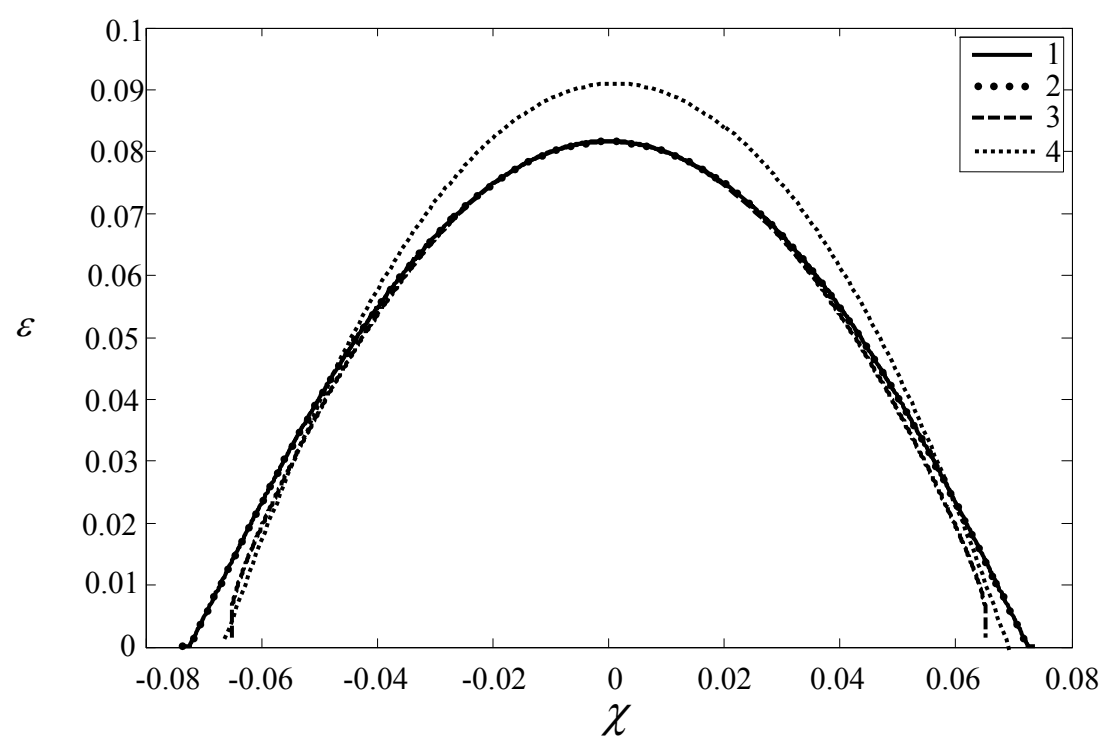

Fig. 8 Profiles of the dissipation rate coefficient of turbulent energy: simulations: $1-N=1000, P_{0}=10 ; 2-N=400, P_{0}=50$; 3 - solution 3.4, 4 - approximate analytical solution.

$\sigma_{k}, c_{\mu}, \sigma_{\varepsilon}, c_{\varepsilon 1}, c_{\varepsilon 2}$, if there is a need to change one, or several coefficients to specify data of experiments, or 3D simulations for the particular flow.

In the present work, we obtained $\beta=0.116$ in $2 \mathrm{D}$ simulations with coefficients from paper [1], while the solution of self-similar equations gives $\beta=0.11$. The linear fitting for experimental data leads to $\beta=0.104$. The averaged 3D ILES results on finer computational grids are also close to the results obtained in the present work. Thus, the k- $\varepsilon$ model coefficients taken in [1] provide a satisfactory description of a plane shear layer of mixing and are preferable, in comparison with the standard coefficients, for describing the gravitational mixing in a plane interfacial layer and a plane light (heavy) layer, as well as the decay of homogeneous isotropic turbulence.

\section{References}

[1] Guzhova, A. R., Pavlunin, A. S. and Statsenko. V. P. 2005. "Specification of Constants for the $k-\varepsilon$ Model of Turbulence Based on Results of the Direct Numerical Simulation for the Simplest Turbulent Flows and Measurements." VANT. ser. TAP 3: 37-48.

[2] Andronov, V. A., Zhidov, I. G., Meshkov, E. E., Nevmerzhitskii, N. V., Nikiforov, V. V. and Razin, A. N. et al. 1995. "Computational and Experimental Studies of
Hydrodynamic Instabilities and Turbulent Mixing." (Review of VNIIEF efforts). LA-12896. CIC-14 Report Collection, Los Alamos National laboratory.

[3] Bakhrakh, S. M., Zhmailo, V. A., Statsenko, V. P. and Yanilkin. Yu. V. 1983. "Numerical Simulation of Turbulent Mixing in Shear Flows." Numerical Methods of Continuum Mechanics 14: N2.

[4] Zhmailo, V. A., Stadnik, A. L., Statsenko, V. P. and Yanilkin, Yu.V. 1995. "Direct Numerical Simulation of Turbulent Mixing in Shear Flows." $5^{\text {th }}$ International Workshop on the Physics of Compressible Turbulent Mixing, Stony Brook (USA),

[5] Zhmailo, V. A., Sin'kova, O. G., Statsenko, V. P. and Yanilkin, Yu. V. 2004. "3D Direct Numerical Simulation of Shear Turbulent Mixing." VANT, ser. TAP 3: 23-39.

[6] Spenser, B. W. and Jones, B. G. 1971. "Statistical Investigation of Pressure and Velocity Fields in the Turbulent Two-Stream Mixing Layers." -AIAA Paper. 613.

[7] Rodi, W. A. 1975. "Review of Experimental Data of Uniform Density Free Turbulent Boundary Layers. Studies in Convection.” Acad. Press, London.

[8] Browand, F. K. and Latigo, B. O. 1979. "Growth of the Two-Dimensional Mixing Layer.” Phys. Fluids 22: 1011.

[9] Oster, D. and Wygnanski, I. 1982. "The Forced Mixing Layer between Parallel Streams." Journal of Fluid Mechanics 123: 91-130.

[10] Hussain, A. K. M. F. and Zedan, M. F. 1978. "Effects of the Initial Condition on the Axisymmetric Free Shear Layer: Effect of the Initial Fluctuation Level ." Phys. Fluids 21 (9): 1100-12

[11] Weisbrot, I., Einav, S. and Wygnanski, I. 1982. "The 
Non-Unique Rate of Spread of the Two-Dimensional Mixing Layer." Phys. Fluids 25 (10): 1691-3.

[12] Balaras, E., Piomelli, U. and Wallace, J. M. 2001. "Self-Similar States in Turbulent Mixing Layers." $J$. Fluid Mech. 446: 1-24,

[13] Rogers, M. M. and Moser, R. D. 1994. "Direct Simulation of a Self-Similar Turbulent Mixing Layer." Phys. Fluids 6: 903-23,

[14] Attili, A. and Bisetti, F. 2012. "Statistics and Scaling of Turbulence in a Spatially Developing Mixing Layer at Re $\lambda=250 . "$ Physics of Fluids 24:035109.

[15] Statsenko, V. P., Velitchko, O. M., Yanilkin, Yu. V. and Zharova, G. V. 1999. "Buoyant Jet Formation." $7^{\text {th }}$ International Workshop on the Physics of Compressible Turbulent Mixing, St.-Petersburg, Russia.

[16] Launder, B. E. and Spalding, D.B. 1974. "The Numerical Computation of Turbulent Flows". Comp. Meth. In Appl. Mech. And Eng. 3: 269-89.

[17] Rodi, W. 1979. "Influence Buoyancy and Rotation on Equations for the Turbulent Length Scale". Proc. 2nd Symp. on Turbulent Shear Flows.

[18] El Tahry, S. H. 1983. "k- $\varepsilon$ Equation for Compressible Reciprocating Engine Flows.” AIAA, J. Energy 7 (4): 345-53.

[19] Llor, A. 2005. "Statistical Hydrodynamic Models for Developed Mixing Instability Flows." Lect. Notes Phys. Springer, Berlin Heidelberg.

[20] Landau L.D., Lifshitz E.M. Continuum Mechanics. - M.: GITTL, 1953.

[21] Paavo. S. 1976. "Two-Point Turbulent Measurements Downstream of a Heated Grid.” Phys. Fluids 19: 1876.

[22] Tennekes H. 1973. "Similarity Laws and Scale Relations in Planetary Boundary Layers." AMS Workshop on micrometeorology. Science Press, Boston.

[23] Read, K. I. 1984. "Experimental Investigation for Turbulent Mixing by Rayleigh-Taylor Instability." Physica D12, 45.

[24] Kucherenko, Yu. A., Shibarshov, L. I., Chitaikin, V. I., Balabin, S. I. and Pylaev, A. P. 1991. "Experimental Study of Asymptotic Stage of the Gravitational Turbulent Mixing Self-Similar Mode." In Proceedings of the 3rd International Workshop on Physics Compressible Turbulent Mixing.

[25] Linden, P. F., Redondo, J. M. and Youngs, D. L. 1994. "Molecular Mixing in Rayleigh-Taylor Instability." $J$. Fluid Mech. 265: 97-124.

[26] Kucherenko, Yu. A., Shestachenko, O. E., Piskunov, Yu. A., Sviridov, E. V., Medvedev, V. M. and Baishev, A. I. 2001. "Experimental Investigations of the Self-Similar Regime of Different-Density Gas Mixing in the Earth Gravity Field.” Presentation at VI Zababakhin Scientific
Readings, Snezhinsk, Russia.

[27] Dimonte, G. and Schneider, M. 2000. "Density Ratio Dependence of Rayleigh-Taylor Mixing for Sustained and Impulsive Acceleration Histories." Physics of Fluids 12 (2): 304-21.

[28] Anisimov, V. I., Kozlovskikh, A. S. and Baban, S. A. 2003. "Analysis of Results for Experiments on Turbulent Mixing with Moderate Reynolds Numbers in the Earth Gravity Field." Presentation at VI Zababakhin Scientific Readings, Snezhinsk, Russia.

[29] Olson, D. H. and Jacobs, J. W. 2009. "Experimental Study of Rayleigh-Taylor Instability with a Complex Initial Perturbation." The Physics of Fluids 21: 034103.

[30] Youngs, D. L. 1994. "Numerical Simulation of Mixing by Rayleigh-Taylor and Richtmyer-Meshkov Instabilites." Laser and Particle Beams 12 (4): 725-50.

[31] Zhmailo, V. A., Stadnik, A. L., Statsenko, V. P. and Yanilkin, Yu. V. 1996. "Direct Numerical Simulation of Gravitational Turbulent Mixing." VANT. Ser. TAP. 1-2: 29-37.

[32] Sin'kova, O. G., Stadnik, A. L., Statsenko, V. P., Yanilkin, Yu. V. and Zhmailo, V. A. 1997. "ThreeDimensional Direct Numerical Simulation of Gravitational Turbulent Mixing." In Proceeding of $6^{\text {th }}$ International Workshop on the Physics of Compressible Turbulent Mixing. Marseille, France.

[33] Yanilkin, Yu. V., Statsenko, V. P., Rebrov, S.V., Sin'kova, O. G. and Stadnik, A. L. 2001. "Study of Gravitational Turbulent Mixing at Large Density Differences Using Direct 3D Numerical Simulation." Report to $8^{\text {th }}$ International Workshop on the Physics of Compressible Turbulent Mixing : $8^{\text {th }}$ IWPCTM, Pasadena, CA, USA.

[34] Dimonte, G., Dimits, A., Weber, S. and Youngsy, D. L. et. al. 2001. "A Comparison of High-Resolution 3D Simulations of Turbulent Rayleigh-Taylor Instability: Alpha-Group Collaboration." Report to $8^{\text {th }}$ International Workshop on the Physics of Compressible Turbulent Mixing: $8^{\text {th }}$ IWPCTM, Pasadena, CA, USA.

[35] Weber, S. V., Dimonte, G. and Marinak, M. M. 2001. "ALE Simulation of Turbulent Rayleigh-Taylor Instability in 2-D and 3-D: Alpha-Group Collaboration." Report to $8^{\text {th }}$ International Workshop on the Physics of Compressible Turbulent Mixing: $8^{\text {th }}$ IWPCTM, Pasadena CA, USA.

[36] Kucherenko, Yu. A., Balabin, S. I. and Pylaev, A. P. 1993. "Experimental Study of Asymptotic Stage of the Gravitational Turbulent Mixing of Thin Liquid Layers of Different Densities." Report to 4th International Workshop on the Physics of Compressible Turbulent Mixing. Cambridge, England. 


\section{Appendix A: Review of experimental data on shear mixing.}

Consider homogeneous flows coming from a spacer plate at velocities $u_{1}$ and $u_{2}$. It is precisely these tests that have been used to study a plane mixing layer and described, in particular, in papers [9-11]. It is assumed that density is uniform in the problem. Thus, the problem is one-dimensional in coordinates of fluid, but it is time-dependent, because in the self-similar regime the plane mixing layer's width grows linearly with time in proportion to the difference $u_{0}$ between the jet velocity and the surrounding medium velocity

$$
b=\beta \cdot u_{0} \cdot t
$$

Here, according to the definition in [9-11], $b=y_{0.95}-y_{0.1}$ is the TMZ width determined for level $\frac{u_{x}}{u_{0}}\left(z_{0.95}\right)=\frac{u_{x}}{u_{0}}\left(\chi_{0.45}\right)=0.45$ and $\frac{u_{x}}{u_{0}}\left(z_{0.1}\right)=\frac{u_{x}}{u_{0}}\left(\chi_{-0.4}\right)=-0.4, \quad$ or $\quad$ in $\quad$ the spacer plate coordinates: $\frac{\tilde{u}_{x}}{u_{0}}\left(z_{0.95}\right)=0.95$ and $\frac{\tilde{u}_{x}}{u_{0}}\left(z_{0.1}\right)=0.1$. Quantity $\beta$ should be represented using quantity $b^{\prime} \equiv d b / d x=b /\left(x-x_{0}\right)$, which is measured directly in experiments.

With regard to $x-x_{0}=\left(u_{1}+u_{2}\right) / 2 \cdot t$ and $u_{0}=u_{2}-u_{1}$, we obtain

$$
\beta=\frac{b^{\prime}}{2 \lambda}, \text { where } \lambda \equiv \frac{u_{2}-u_{1}}{u_{2}+u_{1}},
$$

as defined in [9]-[11]. In other words, with regard to $m \equiv u_{1} / u_{2}$ the expression for $\beta$ can be written as

$$
\beta=\frac{b^{\prime}}{2} \cdot \frac{(1+m)}{(1-m)}
$$

Paper [9] gives the $b^{\prime}$ values calculated by various authors for different values of $\lambda$. We present this data (see Table $\mathrm{A}_{1}$ ) for $\lambda \tilde{<} 0.54$ (these values are not too large) corresponding to $m \tilde{>} 0.3$.

Table $A_{1}$ Experimental data from [9] for the mixing rate.

\begin{tabular}{lll}
\hline$\lambda$ & $\mathrm{m}$ & $\boldsymbol{\beta}$ \\
\hline 0.1474 & 0.743 & 0.120 \\
0.2470 & 0.604 & 0.071 \\
0.2515 & 0.598 & 0.105 \\
0.2546 & 0.594 & 0.084 \\
0.2553 & 0.593 & 0.095 \\
0.2561 & 0.592 & 0.087 \\
0.3366 & 0.496 & 0.094 \\
0.4316 & 0.397 & 0.085 \\
0.4324 & 0.396 & 0.094 \\
0.4324 & 0.396 & 0.093 \\
0.4430 & 0.386 & 0.112 \\
0.5380 & 0.300 & 0.084 \\
0.5418 & 0.297 & 0.094 \\
\hline
\end{tabular}

Fig. A1 illustrates experimental data in the form of plots for $\beta(m)$ function. Measurement results are approximated with the linear dependence

$$
\beta=0.0196 \cdot m+0.084
$$

With $\mathrm{m}=1$ we obtain $\beta=0.1036$ (see also Fig. A1). The root-mean-square deviation of experimental data from straight line (A1) is $\delta \beta=\left\langle(\langle\beta\rangle-\beta)^{2}\right\rangle^{1 / 2} \approx 0.023$ (here, symbols \langle\rangle mean "averaging"), it is shown in Fig. A1. As one can see, with $m \rightarrow 1$ there 
is a satisfactory agreement with the $2 \mathrm{D}$ simulation results. Remember that our temporal mixing layer studies described in this paper correspond to experimental investigations for the spatially evolving configuration in the limit of $\mathrm{m} \rightarrow 1$.

Paper [9] also presents results of various authors, who measured maximum values of root-mean-square fluctuations of different velocity components (see Table $\mathrm{A}_{2}$ ). The turbulent energy values calculated using these measurement results are also given in this table.

These results agree with all the simulation results presented in the present paper.

Results of 2D simulations and self-similar solutions 3.4 are also illustrated by Fig. A2, which shows the reciprocal of growth rate of the mixing layer's width:

$$
\sigma=\frac{1.855 \Delta x}{\Delta\left(z_{0.1}-z_{0.9}\right)},
$$

where, $\Delta\left(z_{0.1}-z_{0.9}\right)$ is the distance between two points, at which relative velocity $\hat{u}=\left(u-u_{2}\right) /\left(u_{1}-u_{2}\right)$ equals 0.1 and 0.9 .

Similar to [3], the jet length $\left(\mathrm{x}^{\left.-\mathrm{x}_{0}\right)}\right.$ in experiments is assumed to be correspondent to time $\left(\mathrm{t}-\mathrm{t}_{0}\right)$ : $\left(x-x_{o}\right)=\left(u_{1}+u_{2}\right) / 2 \cdot\left(t-t_{o}\right)$. Since $\Delta z=u_{0} \cdot\left(\chi_{0.4}-\chi_{-0.4}\right) \cdot\left(t-t_{o}\right)=2 u_{0} \cdot \chi_{0.4} \cdot\left(t-t_{o}\right)$, we have

$$
\sigma=\frac{1.855 \cdot(1+m)}{4 \cdot(1-m) \cdot \chi_{0.4}}=\frac{1.855}{4 \cdot \lambda \cdot \chi_{0.4}}
$$

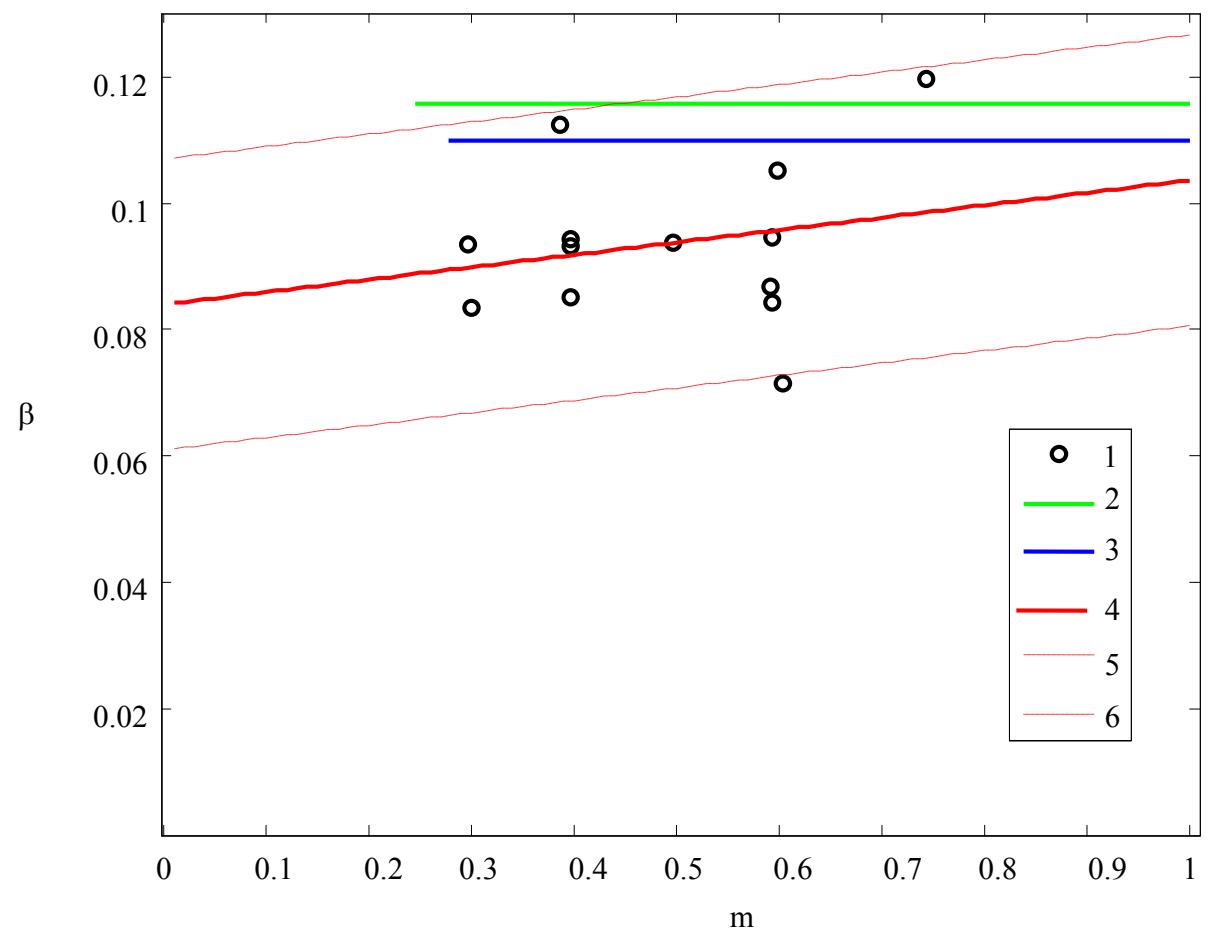

Fig. A1 The mixing rate in a plane layer vs velocity ratio. 1 - experimental data of various authors from paper [9] and linear fitting (4), 5-6 - approximation with root-mean-square deviation; 2 - 2D simulations; 3 - solution of self-similar equations.

Table $\mathrm{A}_{2}$ The root-mean-square fluctuations of velocity components.

\begin{tabular}{lllll}
\hline Authors & $u_{x}^{\prime} / u_{0}$ & $u_{y}^{\prime} / u_{0}$ & $u_{z}^{\prime} / u_{0}$ & $k_{m}$ \\
\hline Spencer 1970 & 0.17 & 0.14 & 0.145 & 0.0348 \\
Yule 1971 & 0.173 & 0.16 & 0.18 & 0.044 \\
Oster, Wygnanski 1982 & 0.18 & 0.153 & 0.145 & 0.0384 \\
\hline
\end{tabular}


where, $m \equiv u_{1} / u_{2}$ and velocities are associated with the coordinate system of a spacer plate, or a nozzle. Similarly, Fig. A2 illustrates data of measurements from [8] within the range of $m$ values corresponding to the range of $\lambda$ values. Points in Fig. A2 show experimental data of various authors from paper [7].

One can see that the calculated curves agree with measurement results. The best agreement is observed at large $m$ values, which correspond to the considered problem to a higher extent.

\section{Appendix B: Review of the direct 3D numerical simulation results for shear mixing.}

Papers $[4,5]$ give the 3D ILES results for different values of $\mathrm{N}_{\mathrm{x}}$ (the number of cells in the flow motion direction). We present this data in Table $\mathrm{A}_{3}$ after additional post-processing efforts. The TMZ width in the papers above, $\Delta\left(z_{0.1}-z_{0.9}\right)$ is the distance between two points, at which relative velocity $\hat{u}=\left(u-u_{2}\right) /\left(u_{1}-u_{2}\right)$ equals 0.1 and 0.9 . However, according to the definitions in $[9,11]$, we take $b=z_{0.95}-z_{0.1}$, i. e. the TMZ width for level $\frac{u_{x}}{u_{0}}\left(z_{0.95}\right)=\frac{u_{x}}{u_{0}}\left(\chi_{0.45}\right)=0.45$ and $\frac{u_{x}}{u_{0}}\left(z_{0.1}\right)=\frac{u_{x}}{u_{0}}\left(\chi_{-0.4}\right)=-0.4$, or in the spacer plate coordinates: $\frac{\tilde{u}_{x}}{u_{0}}\left(z_{0.95}\right)=0.95$ and $\frac{\tilde{u}_{x}}{u_{0}}\left(z_{0.1}\right)=0.1$. In this case, we obtain the values of $\beta=\frac{b}{u_{0} \cdot\left(t-t_{0}\right)}$ given in Table $\mathrm{A}_{3}$.

Besides, the post-processing of the 3D ILES results from [12] and DNS results from [13] gives us $\beta=0.064 \div 0.08$ and $\beta=0.077$, respectively. Similar to our simulations, their simulations were performed for a temporal mixing layer. Simulations with the 3D DNS method in [14] were performed for a spatially evolving configuration. Regarding that $m$ in [14] equals $u_{1} / u_{2}=1 / 3$, we have $\beta=0.5 b^{\prime}(1+m) /(1-m)=b^{\prime}$. Here, $b^{\prime} \equiv d b / d x=\Delta y / \Delta x=r_{\theta} \cdot \Delta y^{+}, r_{\theta}=\Delta \theta / \Delta x=0.0168$ according to [14]. $\Delta y^{+}=\Delta y / \Delta \theta$ is found using the velocity profiles from [14] and, as a result, we obtain $\beta=0.086 \div 0.093$ for data in [14]. These results agree with the data in [4-5].

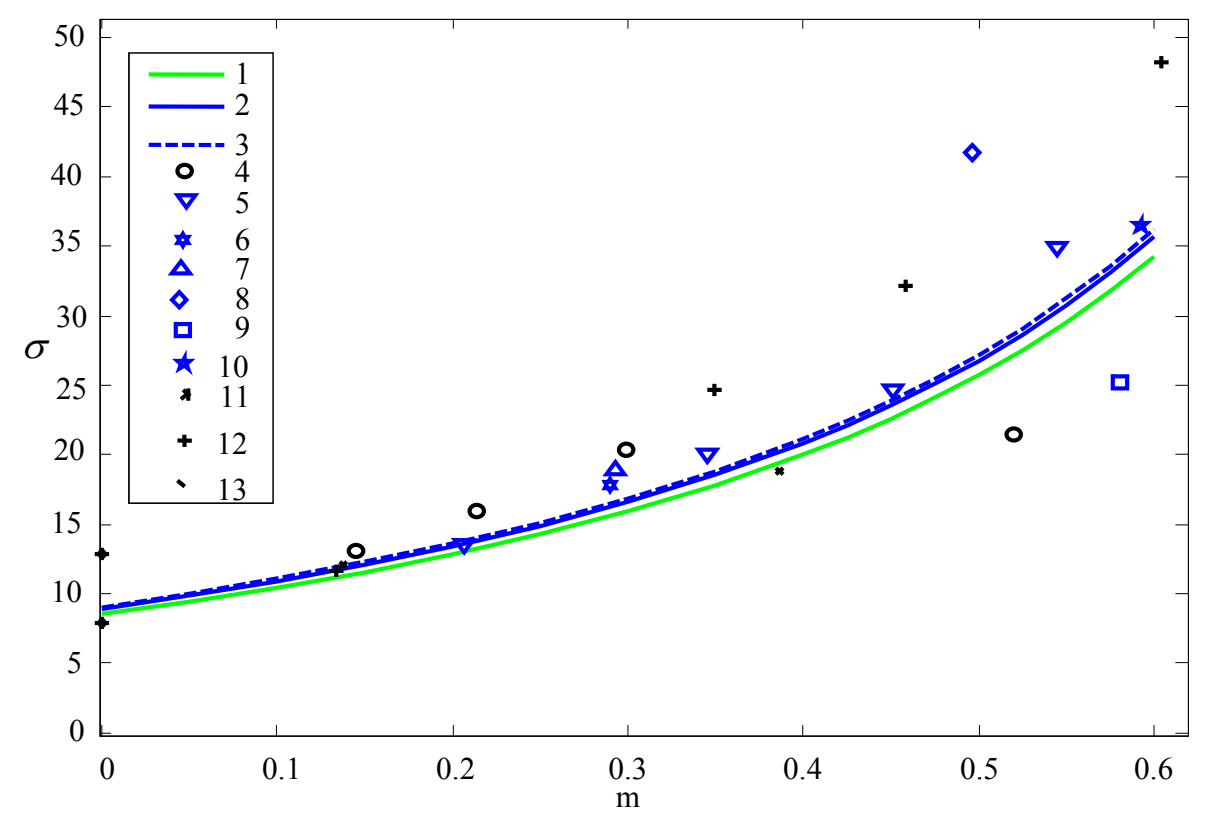

Fig. A2 Quantity $\sigma$ as a function of time in the problem of a shear mixing layer. Present paper: 1 - 2D simulations; 2 -

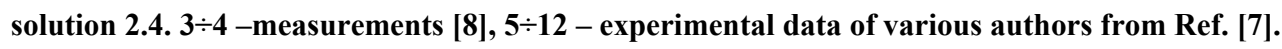


Table $A_{3}$ The integral 3D ILES values.

\begin{tabular}{llllll}
\hline Option No. & Number of cells & $\mathrm{L}_{\mathrm{z}}$ & $\mathrm{L}_{\mathrm{x}}$ & $\beta$ & $k_{m}$ \\
\hline 1 & $100^{3}$ & 1 & 1 & $0.077 \div 0.09$ & $0.033-0.037$ \\
2 & $100^{3}$ & 1 & 1 & $0.07 \div 0.1$ & 0.027 \\
3 & $100^{3}$ & 1 & 1 & 0.096 & 0.036 \\
4 & $150^{2} \times 200$ & 2 & 1.5 & $0.1 \div 0.1075$ & $0.038 \div 0.041$ \\
5 & $200^{2} \times 400$ & 2 & 1 & $0.098 \div 0.1$ & $0.038 \div 0.04$ \\
6 & $300^{2} \times 400$ & 2 & 1.5 & $0.086 \div 0.1$ & $0.033 \div 0.035$ \\
7 & $300^{2} \times 400$ & 2 & 1.5 & $0.13-0.17$ & 0.05 \\
\hline
\end{tabular}

Fig. A3a illustrates the data from [4-5] as a plot of function $\beta\left(N_{x}\right)$. The linear fitting of this data is

$$
\beta=1.665 \cdot 10^{-4} \cdot N_{x}+0.07115 \text {. }
$$

With $\mathrm{N}_{\mathrm{x}}=300$ we have $\beta=0.121$ (see also Fig. A3). The root-mean-square deviation from straight line (A2) is $\delta \beta=\left\langle(\langle\beta\rangle-\beta)^{2}\right\rangle^{1 / 2} \approx 0.024$, it is shown in Fig. A3. One can see a satisfactory agreement with the $2 \mathrm{D}$ simulation results and solutions of self-similar equations.

Fig. A3b illustrates 3D simulation results for the maximum turbulent energy in the form of $k_{m}\left(N_{x}\right)$ function plots. The linear fitting of this data is

$$
k_{m}=2.873 \cdot 10^{-5} \cdot N_{x}+0.03178
$$

In this case, the root-mean-square deviation of results from straight line (A3) is $\delta k_{m}=\left\langle\left(\left\langle k_{m}\right\rangle-k_{m}\right)^{2}\right\rangle^{1 / 2} \approx 0.0055$, it is shown in Fig. A3b. As one can see, there is a satisfactory agreement with all results of 2D simulations and self-similar equation solutions.

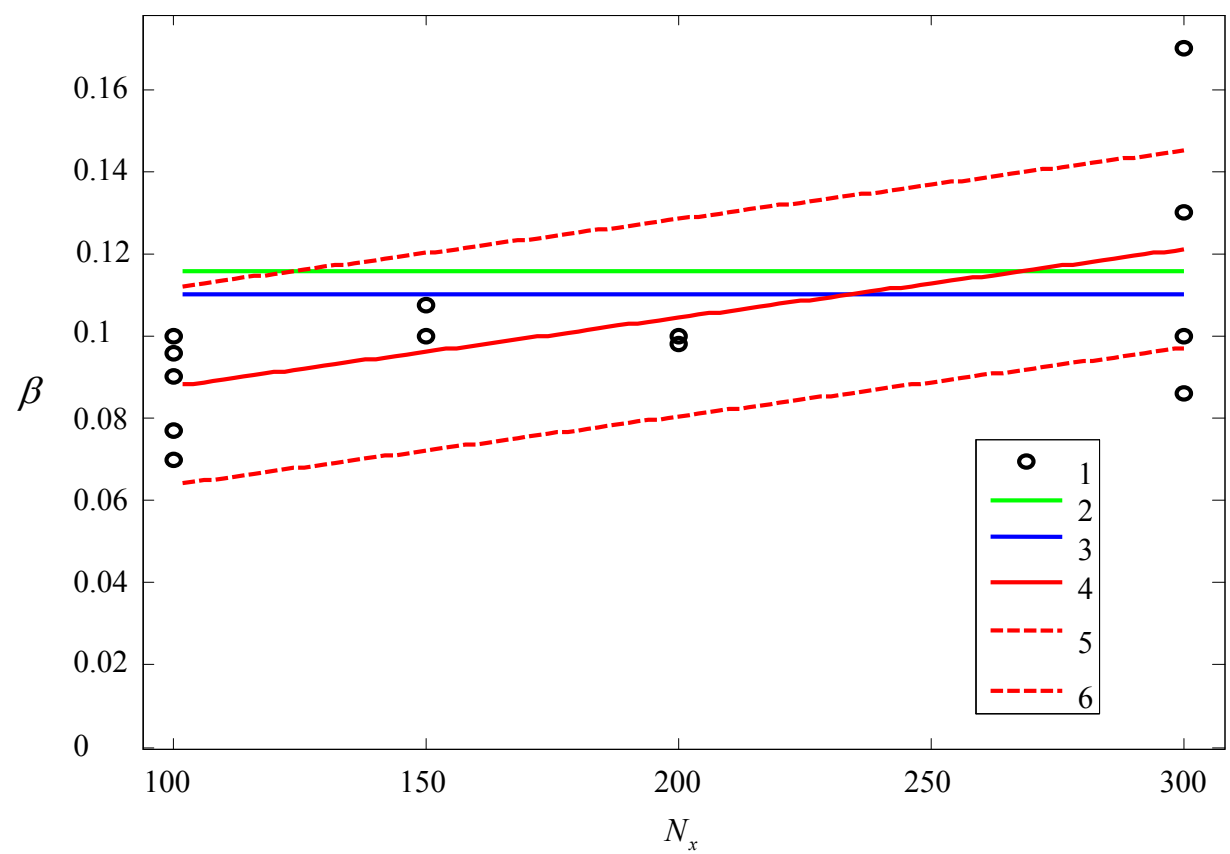

(a) 
Solution of Self-Similar Equations of the k- $\varepsilon$ Model in the Shear

Turbulent Mixing Problem and Its Numerical Simulation

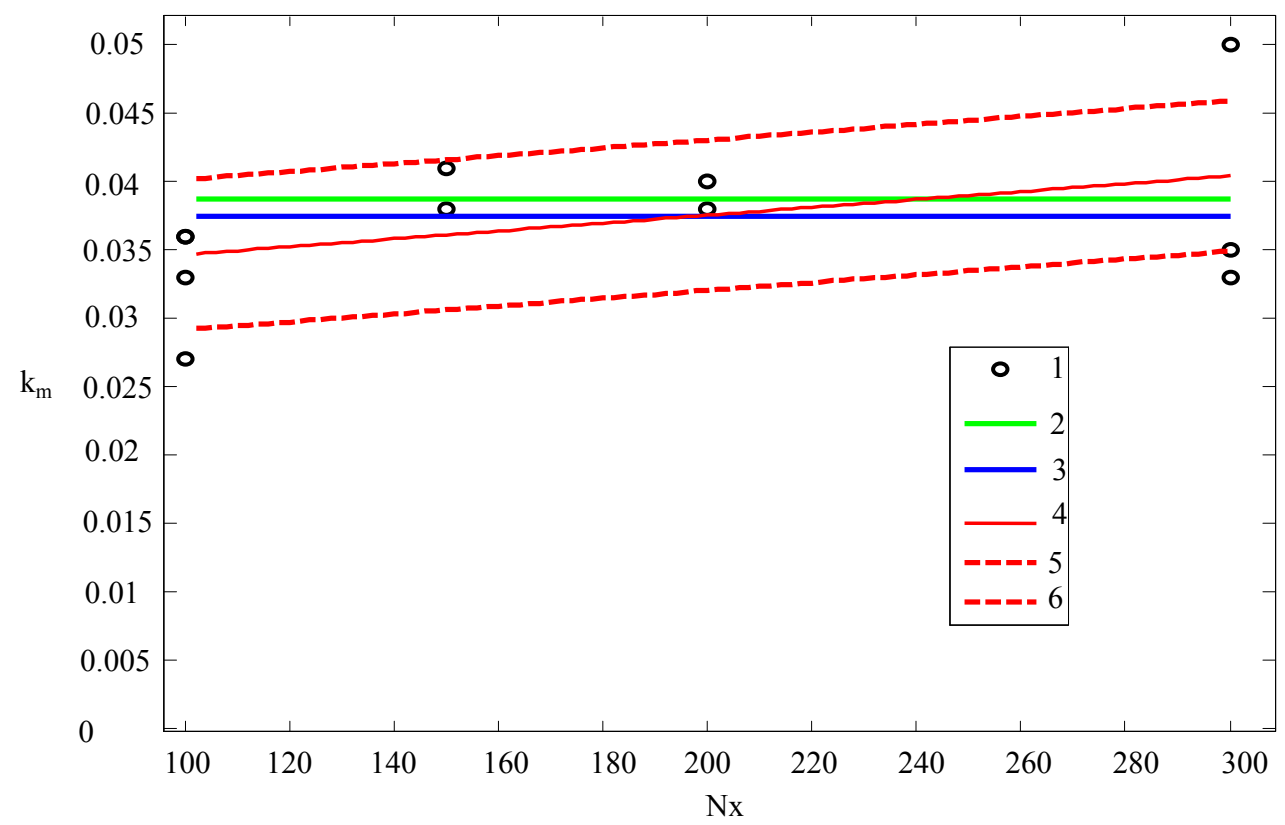

(b)

Fig. A3 The mixing rate of a plane layer (a) and the maximum turbulent energy in TMZ (b) vs. the number of computational cells. 1 - 3D ILES results and linear fitting (4); 5-6 -fitting with RMS deviation; 2 - 2D simulations; 3 solution of self-similar equations. 